\title{
DISPONIBILIDAD DE NITRÓGENO EN CULTIVOS HERBÁCEOS EXTENSIVOS ECOLÓGICOS Y CONVENCIONALES: RELACIÓN CON LA CANTIDAD DE CARBONO
}

\author{
${ }^{\bullet}$ Daniel Vera Avilés ${ }^{1}$, Joan Romanya ${ }^{2}$, Felipe Garcés Fiallos ${ }^{1}$, Freddy Sabando Ávila ${ }^{1}$, Gabriel LiuBa Delfini ${ }^{1}$ y \\ Luis Godoy Montiel \\ ${ }^{1}$ Unidad de Investigación Científica y Tecnológica, Universidad Técnica Estatal de Quevedo, km 7 vía \\ Quevedo - El Empalme, C. P. 73. Mocache,Los Rios, Ecuador. ${ }^{\bullet}$ federic_net@hotmail.com \\ ${ }^{2}$ Departamento de Productos Naturales, Biología Vegetal y Edafología. Universidad de Barcelona. \\ Avda. Joan XXIII s/n 08028 Barcelona, España.
}

\section{RESUMEN}

$\mathrm{S}_{\mathrm{c}}^{\mathrm{c}}$ determinó la disponibilidad de nitrógeno en cultivos herbáceos extensivos, en formas de $\mathrm{NO}_{3}^{-}$, $\mathrm{NH}_{4}^{+}$y NPM (nitrógeno potencialmente mineralizable), en la zona de Huesca (España). En los sistemas agrícolas convencionales se realiza un mayor aporte de nitrógeno $(\mathrm{N})$ que en los ecológicos; en estos, por otro lado, el aporte de materia orgánica es mucho mayor que en los convencionales. La consecuencia lógica sería de esperar un mayor contenido de $\mathrm{N}$ en los suelos de cultivos convencionales, y un mayor contenido de materia orgánica en los suelos de cultivos ecológicos Sin embargo, los resultados de porcentaje de carbono (C) (representativo en materia orgánica) obtenido en este estudio no corroboraron la teoría, porque la principal diferencia se encontró en la gestión de regadío y secano, independientemente de si se trataba de sistemas convencionales o ecológicos. La mayor disponibilidad de nitrógeno se encontró en los suelos de gestión de regadíos ecológicos.

Palabras claves: nitrógeno, carbono, materia orgánica, suelo, sistemas agrícolas.
Abstract

$\mathrm{T}$ he availability of nitrogen in extensive herbaceous crops in the form of $\mathrm{NO}_{3}^{-}, \mathrm{NH}_{4}^{+}$and $\mathrm{PMN}$ (potentially mineralizable nitrogen) in the area of Huesca (Spain) was determined. In conventional farming systems a greater contribution of nitrogen $(\mathrm{N})$ is made than in the ecologic farming, but in these, on the other hand, the contribution of organic matter is much higher than conventional ones. The logical consequence would be to expect a higher content of $\mathrm{N}$ in soils from conventional farming, and increased organic matter content from soils with organic farming. However, the results of percentage of carbon (C) (representative of organic matter) obtained in this study did not corroborate the theory, because the main difference was found in the rainfed and irrigation management, regardless of whether it was conventional systems or ecological. The increased availability of nitrogen was found in soils with organic irrigation management.

Key words: nitrogen, carbon, organic matter, soil, agricultural systems.

\section{INTRODUCCIÓN}

$\mathrm{E}^{\mathrm{n}}$ los sistemas de cultivos ecológicos, la gestión de la fertilidad es uno de los aspectos más importantes que limitan su producción. La agricultura ecológica, también llamada agricultura orgánica en algunos países, basa la condición de la fertilidad del suelo en la materia orgánica y los procesos biológicos del suelo (bacterias fijadoras de nitrógeno). Dado que los organismos del suelo son generalmente heterótrofos, su actividad será especialmente relevante en un contexto de buena disponibilidad de nitrógeno (Lampkin, 1998; Ostegard, 2002).

El nitrógeno es, después del agua, el principal factor limitante para el desarrollo de los cultivos.
Precisamente por esta razón en el periodo entre 1950 y 1990 el uso de fertilizantes nitrogenados se multiplicó por diez, lo cual llevó a un aumento sin precedentes de la productividad en los cereales. Sin embargo, la aplicación de estos fertilizantes $\mathrm{y}$ otras acciones industriales y antrópicas ha alterado las condiciones básicas del ciclo natural del nitrógeno y ha contribuido a la contaminación por nitratos en los ecosistemas terrestres y acuáticos, con graves riesgos para la salud humana (Fernández-Pascual et al., 2002).

Los suelos agrícolas se caracterizan por contener menores concentraciones de carbono orgánico que los suelos forestales. Además, la intensificación

Recibido: 9-Julio-2011. Recibido en forma corregida: 13-Septiembre- 
de la gestión de los suelos agrícolas que ha ocurrido en Europa durante la segunda mitad del siglo XX ha resultado en una disminución destacable de la concentración de carbono del suelo (Maljean et al., 2004). Loveland y Webb (2003), en una revisión sobre los niveles críticos de materia orgánica en suelos agrícolas del área templada, sugirieron que un contenido de carbono de $1 \%$ podría representar el umbral por debajo del cual el funcionamiento del sistema suelocultivo podría quedar comprometido, incluso cuando se suministraran los fertilizantes minerales adecuados.

Conviene destacar que en la revisión de Loveland y Webb (2003), no se mencionan casos por debajo de este valor que, según ellos, en cultivos de la zona templada, se mantiene solamente a partir de las adiciones anuales de materia orgánica por parte del cultivo.

De manera general, el contenido de materia orgánica del suelo se relaciona íntimamente con las condiciones climáticas y ambientales de cada zona. Por ello, para analizar los factores de variación del carbono del suelo en suelos agrícolas, conviene considerar los factores clima, el tipo de cultivo y el modelo de gestión. En agricultura ecológica, el tipo de cultivo y el modelo de gestión suelen estar imbricados, dado que en este modelo de gestión a menudo se utilizan rotaciones y policultivos (Romanyà et al., 2007). El objetivo de esta investigación es cuantificar la disponibilidad de nitrógeno en campos de cultivo sometidos a diferentes prácticas agrícolas, para así averiguar si el tipo de gestión (convencional y ecológica), así como la disponibilidad de agua (regadío y secano) generan cambios significativos en el contenido de $\mathrm{N}$ mineralizable del suelo.

\section{Materiales y Métodos}

\section{Área de muestreo}

$\mathrm{E}$ sta investigación fue realizada en la comarca de Los Monegros, concretamente en Sariñena-Castelflorite, Huesca, España, donde los cultivos herbáceos verdes han crecido por lo menos durante los últimos 40 años. Durante este tiempo, algunas de las áreas han sido regadas por inundación, mientras que el resto de las tierras agrícolas se ha mantenido en secano. El clima es de tipo semiárido mediterráneo, caracterizado por una temperatura media anual de $14.2^{\circ} \mathrm{C}$, precipitación $442.9 \mathrm{~mm}$, a una altitud de $300 \mathrm{msnm}$. Los suelos son alcalinos, con $\mathrm{pH}$ de 8.1 a 9.2, y de texturas arcilloarenosas (Romanyà y Rovira, 2007).

Los suelos de secano ecológico y convencional realizan una rotación cebada-barbecho. En los suelos convencionales de regadío se lleva a cabo un monocultivo de trigo, mientras que en los suelos con manejo orgánico irrigados se realiza una rotación de trigo, avena y guisantes. En el momento del muestreo, todos los sitios de riego fueron sembrados con trigo y todos los sitios de secano con cebada.

La agricultura ecológica evita el uso de pesticidas y fertilizantes sintéticos. Los suelos manejados orgánicamente reciben una aplicación de estiércol de aves de corral: $5 \mathrm{Mg} \mathrm{ha}^{-1}$ cada dos años para las tierras de secano, y $10 \mathrm{Mg} \mathrm{ha}^{-1}$ año $^{-1}$ para los suelos de regadío.

La fertilización con $\mathrm{N}$ de los campos convencionales consistió en 100 y $120 \mathrm{~kg} \mathrm{~N}^{-1}$ año $^{-1}$ de Urea o un fertilizante similar en los suelos de secano, y entre 140 y $160 \mathrm{~kg} \mathrm{~N} \mathrm{ha}^{-1}$ año $^{-1}$ en suelos de regadío. En los campos con gestión ecológica no podemos precisar la cantidad de $\mathrm{N}$ añadido con el estiércol de aves de corral, al no disponer del contenido de $\mathrm{N}$ de este fertilizante.

\section{Recolección de muestras para posterior análisis}

Para la recolección de las muestras de suelo, se seleccionaron ocho campos que habían sido gestionados bajo un sistema orgánico a lo largo de 18 años (cuatro irrigados regularmente y cuatro de secano), y ocho campos con sistema convencional (cuatro irrigados y cuatro de secano). Se muestrearon tres puntos por campo. Cada suelo muestreado fue dividido en dos capas $0-10$ y $10-20 \mathrm{~cm}$ de profundidad, totalizando 96 muestras diferentes, usando una sonda cilíndrica para la recolección de las muestras. Todas las muestras fueron secadas al aire bajo sombra y tamizadas en malla de $2 \mathrm{~mm}$. Una submuestra fue molida para los análisis químicos totales.

\section{Análisis de formas de nitrógeno mineral en las muestras}

La extracción de nitrógeno mineral $\left(\mathrm{NO}_{3}^{-}{ }^{-}\right.$ $\mathrm{NH}_{4}^{+}$) en las muestras obtenidas se realizó siguiendo el protocolo de Keeney y Nelson (1982). Se pesó cinco gramos de tierra seca y $12.5 \mathrm{~mL}$ de agua de $\mathrm{KCl} 2 \mathrm{M}$, a continuación se agitaron las muestras durante una hora con la ayuda de un agitador rotatorio y posteriormente se recuperó el líquido por filtración. En el extracto se cuantificó el amonio por el método del azul de indofenol y el nitrato por absorción directa en el ultravioleta, a $220 \mathrm{~nm}$. Para el nitrato, los cálculos se llevaron a cabo con la absorbancia corregida: ABS220- $(2 \times$ ABS275) (Slanina et al., 1976). 
Determinación de nitrógeno potencialmente mineralizable (NPM).

Se analizó el NPM como índice de capacidad de mineralización de $\mathrm{N}$ del suelo. El método se basó en una incubación anaeróbica a $40^{\circ} \mathrm{C}$ durante siete días. El cálculo del nitrógeno potencialmente mineralizable se realizó restando el amonio previo a la incubación del amonio total tras la incubación.

\section{Determinación de carbono orgánico}

La medición del porcentaje de carbono orgánico se llevó a cabo mediante el método de oxidación con dicromato de Walkley y Black (1934).

\section{Análisis estadístico}

En el experimento se estudiaron las formas de nitrógeno mineral (nitrato y amonio) así como el tipo de gestión (convencional y ecológico) y la disponibilidad de agua (regadío y secano). El diseño estadístico utilizado fue completamente al azar con tres repeticiones, aplicando una ANOVA de dos factores (uso: convencional versus ecológico; y disponibilidad de agua: riego versus secano), con interacciones. Se utilizó el programa estadístico SPSS versión 14.0 (SPSS, 2005). Para la comparación de medias se realizó la prueba de rangos múltiples de Tukey al $\mathrm{p}<0.05$ de significación. Los resultados finales se expresaron en $\mathrm{mg} \mathrm{kg}^{-1}$ en el caso del nitrógeno y en porcentaje en el caso del carbono.

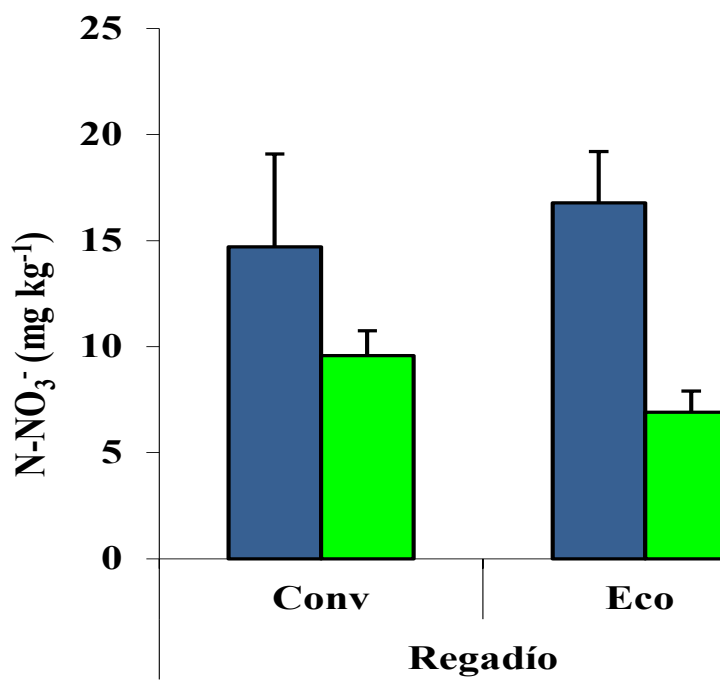

\section{Resultados y Discusión}

Disponibilidad y formas de nitrógeno en el suelo $\left(\mathrm{NO}_{3}^{-}, \mathrm{NH}_{4}^{+}\right)$y NPM

Se determinó que la disponibilidad de agua (regadío y secano) y la profundidad (0-10 y 10-20) afectaron significativamente al contenido de nitrato del suelo (Fig. 1), la elevada solubilidad del nitrato sobre todo se reflejaría en un mayor lavado, del horizonte superior $(0-10 \mathrm{~cm})$ al inferior $(10-20 \mathrm{~cm})$. Así, habría que esperar que en suelos irrigados la diferencia entre el contenido de nitrato en superficie y en profundidad fuera menor. Sin embargo observamos lo contrario. En suelos de secano la concentración de nitrato a $0-10 \mathrm{~cm}$ y a $10-20 \mathrm{~cm}$ es muy parecida; en cambio es precisamente en suelos irrigados donde el nitrato se concentra más en superficie. La elevada temperatura y evapotranspiración de la comarca (zona semiárida) pueden haber arrastrado el nitrato a la superficie, lo que demuestra el papel crucial de los factores climáticos y en especial a la disponibilidad de agua en el suelo, concordando con los resultados de Grist (1982). Las mayores cantidades de nitrato $\left(\mathrm{NO}_{3}^{-}\right)$en el suelo se observaron en campos de regadío; esto se debe a su alta movilidad en el suelo (Lampkin, 1998) gracias a que la solubilidad de los compuestos nitrogenados es alta. Al evaluar el contenido de amonio (Fig. 2), el análisis detectó diferencias estadísticas a nivel 0.05 para riego e interacción. Las parcelas de regadío ecológico presentaron el mayor promedio de contenido de amonio, superior estadísticamente al del resto de parcelas, mientras que también se observa un incremento de

Figura 1. Contenido de nitrato total en muestras de tierras extraídas de las parcelas indicadas, en dos profundidades. 


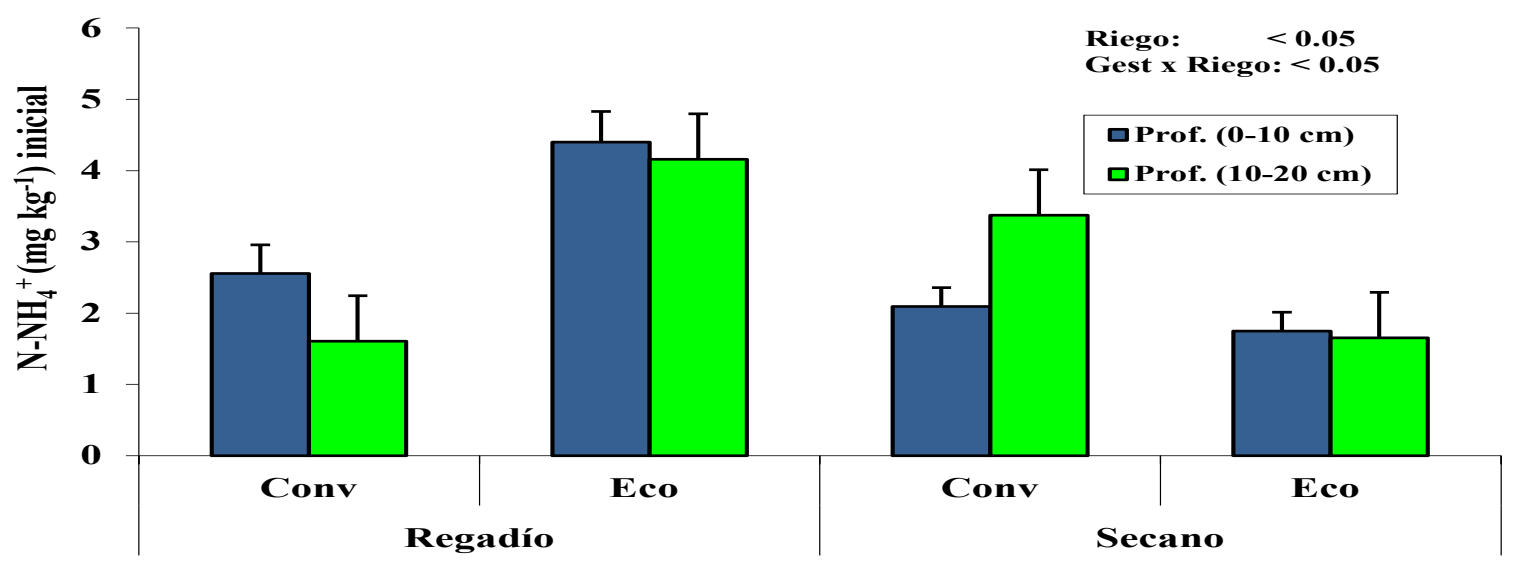

Figura 2. Contenido de amonio total en muestras de tierras extraídas de las parcelas indicadas, en dos profundidades.

amonio en la profundidad 10-20 en la parcela de secano convencional de acuerdo a la otra profundidad. La fijación de amonio por materia orgánica y arcillas debería suponer un freno al lavado de amonio lo cual concuerda con Lampkin (1998), quien afirma que el nitrógeno está en forma de compuestos orgánicos integrados en la materia orgánica del suelo, prácticamente inasimilable por las plantas, y en formas inorgánicas disponibles para las plantas como iones amonio $\left(\mathrm{NH}_{4}^{+}\right)$y nitrato $\left(\mathrm{NO}_{3}^{-}\right)$. Los iones amonio son relativamente estables, pues la materia orgánica y las partículas de arcilla lo adsorben. Las bacterias, los hongos y otros microorganismos edáficos son responsables de una u otra forma de la transformación del nitrógeno. Las actividades de estos microorganismos y por lo tanto la forma de nitrógeno en el suelo, se ven afectadas profundamente por el laboreo del suelo, las aportaciones orgánicas, las rotaciones de los cultivos y los exudados de raíces.
Al analizar el contenido de nitrógeno potencialmente mineralizable (método incubación) se observaron diferencias significativas a nivel 0.05 tanto para riego, profundidad e interacción (gestión $\mathrm{x}$ riego). Las parcelas ecológicas de regadío presentaron el mayor contenido de NPM, superior estadísticamente al de las demás parcelas. El NPM obtenido en superficie (0-10 $\mathrm{cm}$ ) fue claramente superior al obtenido en profundidad $(10-20 \mathrm{~cm})$ (Fig. 3). Esto ya fue observado por Rodríguez y Silva (1984), que sostienen que en el estrato superficial del suelo $(0-10 \mathrm{~cm})$ de praderas naturales y campos de cereales se desarrolla un profuso sistema radicular que aportaría, junto a los residuos vegetales de la parte aérea, cantidades importantes de nitrógeno potencialmente mineralizable. En comparación con estos aportes, los recibidos por el estrato inferior del suelo $(10-20 \mathrm{~cm})$ serían mucho menores.

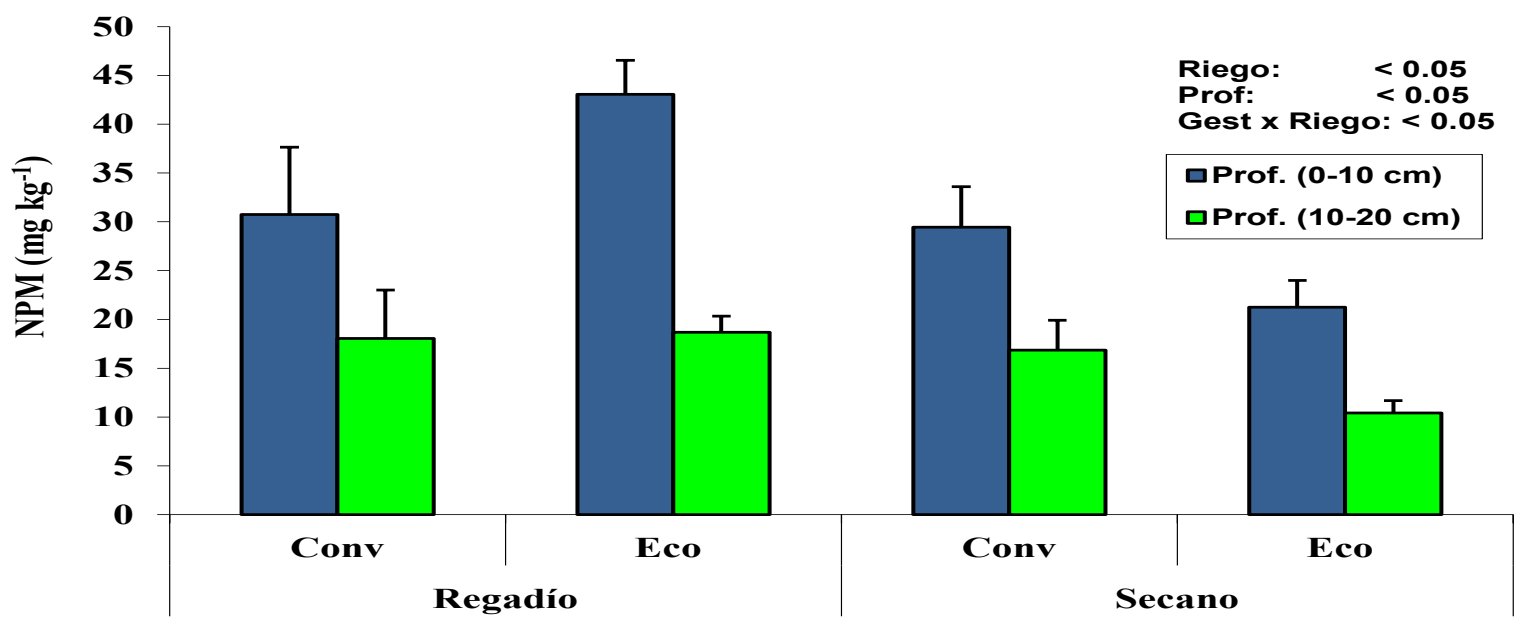

Figura 3. Nitrógeno potencialmente mineralizable (método incubación) en muestras de tierras extraídas de las parcelas indicadas, en dos profundidades. 


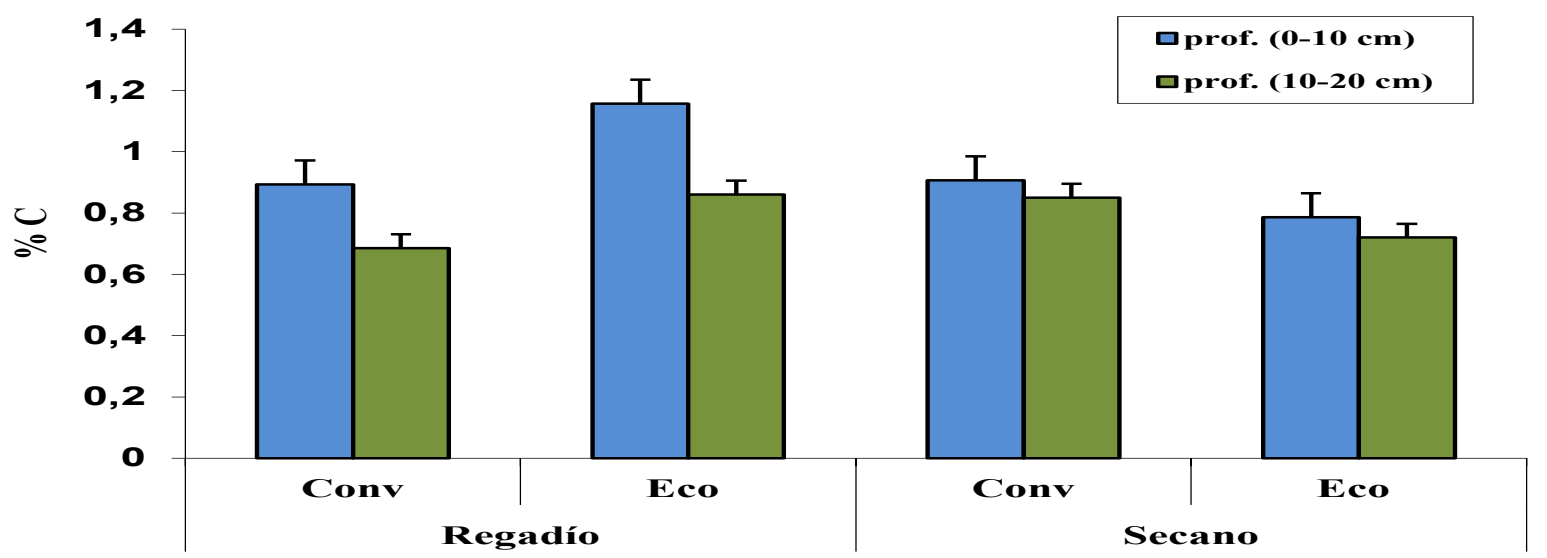

Figura 4. Porcentaje de carbono (C) en parcelas ecológicas y convencionales de secano y regadío en dos profundidades estudiadas.

Porcentaje de carbono (C) para cada profundidad en estudio

Se observó una disminución del porcentaje de carbono con la profundidad en todos los casos. El porcentaje de carbono analizado en el horizonte superficial de las parcelas ecológicas de regadío fue significativamente mayor al encontrado en las parcelas ecológica de secano (Fig. 4). Se preveía que en las parcelas sometidas a agricultura ecológica la materia orgánica del suelo no sólo sería más abundante, sino también más fácilmente mineralizable por la microflora. Sin embargo los resultados obtenidos en este estudio no coinciden completamente con lo esperado, ya que en conjunto no puede afirmarse que la agricultura ecológica resulte en un aumento del carbono del suelo: se observa este aumento en los sistemas irrigados, pero no en los suelos de secano.

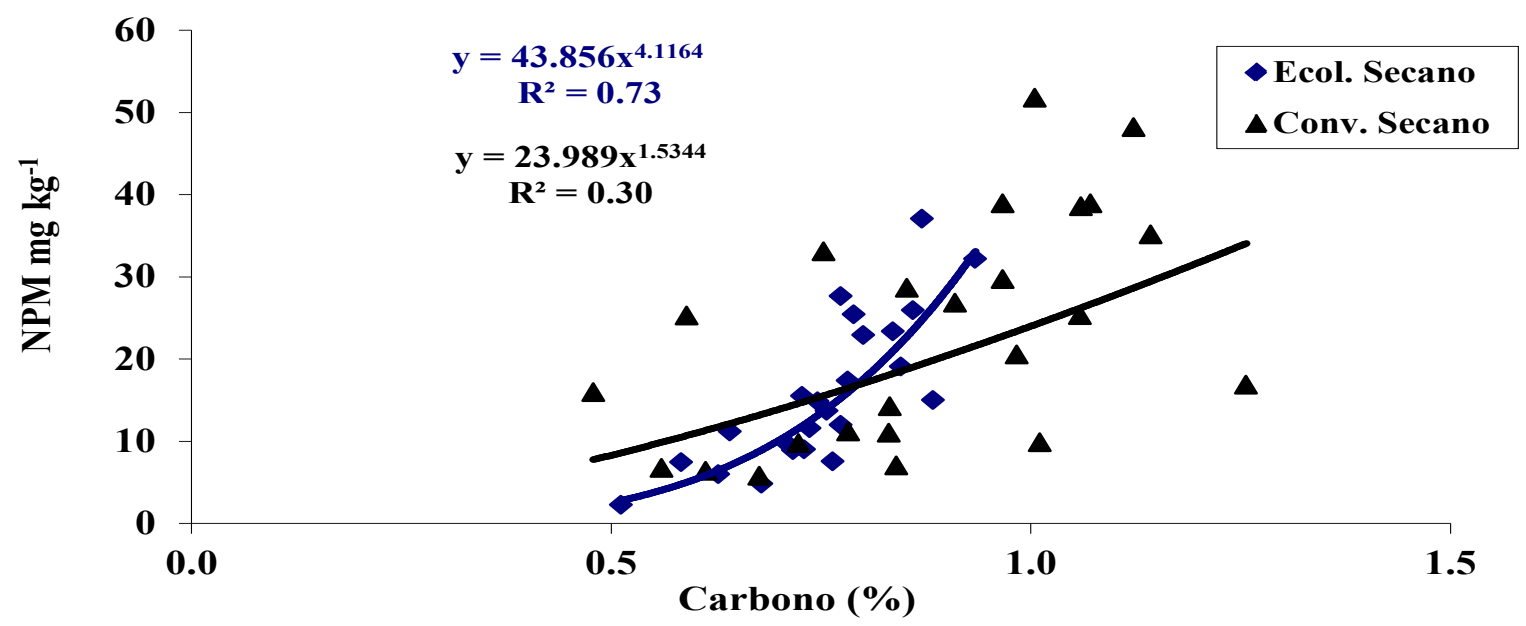

Relación materia orgánica - disponibilidad de nitrógeno

Los sistemas convencionales se caracterizan por la aplicación de altas cantidades de fertilizantes, por lo que resultaría lógico encontrar mayor disponibilidad de N en estas parcelas. No obstante, esto sólo se observó para las parcelas en secano, en las cuales el NPM fue superior en las convencionales que en las ecológicas. En los sistemas de regadío, los niveles de NPM fueron superiores en las parcelas ecológicas (Fig. 3).

El análisis de correlación realizado (Figura 5 y 6), señala una alta correlación positiva $(\mathrm{P}<0.01)$ entre el contenido de nitrógeno potencialmente mineralizable y el porcentaje de carbono. Estas correlaciones podrían ser válidas para suelos similares.

La cantidad y diversidad de microorganismos presentes en el suelo están estrechamente relacionadas con la calidad del suelo donde se hallan y con la calidad

Figura 5. Correlación entre el porcentaje de carbono y nitrógeno potencialmente mineralizable $\left(\mathrm{mg} \mathrm{kg}^{-1}\right)$; en parcelas ecológicas y convencionales de secano. 


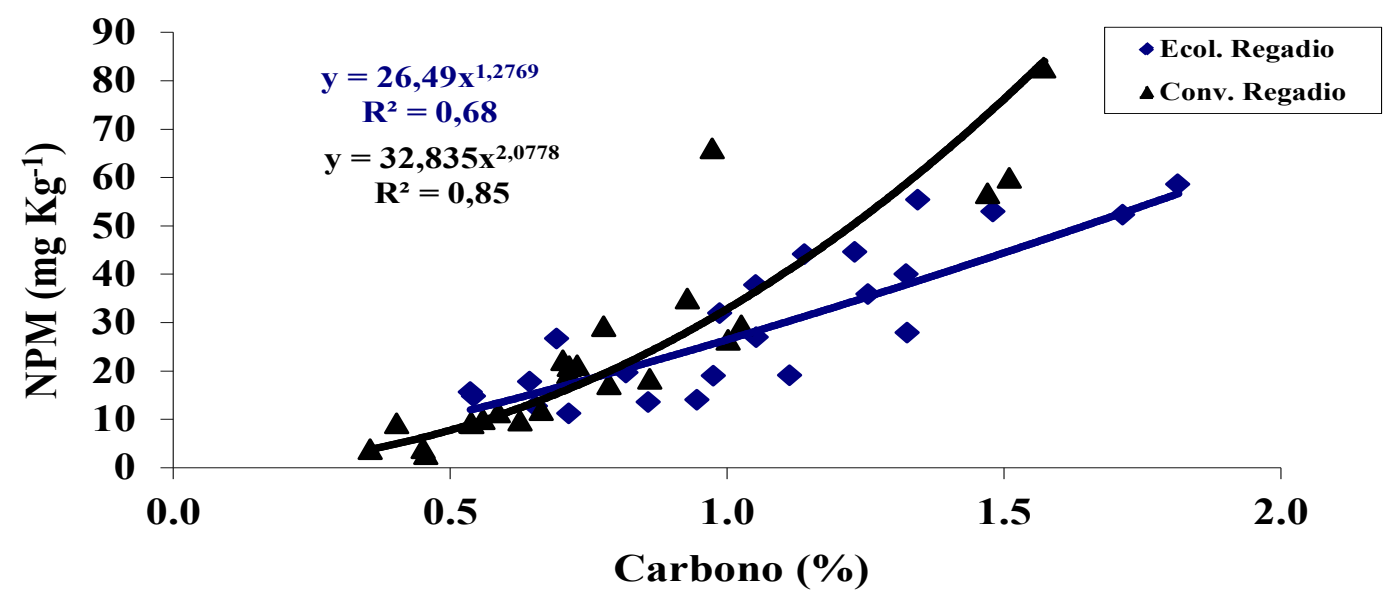

Figura 6. Correlación entre el porcentaje de carbono y nitrógeno potencialmente mineralizable (mg kg-1); en parcelas ecológicas y convencional de regadío.

de su estructura. Es por eso que en cultivos ecológicos, donde reina el aporte de materia orgánica y el uso de técnicas favorables para la estructuración del suelo, es esperable una mayor riqueza de microorganismos y una mayor actividad de éstos, en comparación con los suelos de cultivos convencionales. Coinciden con esta visión los resultados de Puig (1979), quien observó que en suelos fértiles el nitrógeno aumentaba con el tiempo, lo que sugería que los microorganismos eran los agentes causantes de este enriquecimiento. Por su lado, Lacasta y Meco (2005), manifiestan que el uso de rotaciones con leguminosas y aporte de residuos de cosecha aumenta los niveles de materia orgánica, restaurando el equilibrio mineralización-humificación, favoreciendo el desarrollo de organismos simbiontes, generando biodiversidad y vida en el suelo, disminuyendo los efectos de contaminación por lixiviado, y haciendo viable económica y ambientalmente estos agrosistemas de secano y regadío.

\section{Conclusiones}

T as parcelas de regadío ecológico presentaron los mayores promedios de disponibilidad de $\mathrm{N}$ y superioridad estadística respecto a los obtenidos con las parcelas restantes.

La rotación de cultivos y el paso de una agricultura convencional a una agricultura ecológica se traducen en un aumento de la materia orgánica del suelo en condiciones de regadío. Por el contrario, en condiciones de secano el paso de una agricultura convencional a una agricultura ecológica parece producir una disminución de contenido de materia orgánica del suelo.

Se demuestra una alta correlación positiva entre la materia orgánica $(\% \mathrm{C})$ y el nitrógeno potencialmente mineralizable (NPM), tanto para la condiciones de secano como para las condiciones de regadío. Esta relación puede estar influida por los manejos agrícolas, como la cantidad, el momento de aplicación y la forma química de los fertilizantes, la irrigación, la utilización de cultivos de cobertera, la incorporación de residuos vegetales y el laboreo (Reigosa et al., 2005)

\section{LITERATURA CITADA}

Fernández-Pascual M., N. De María, M. R. De Felipe. 2002. Fijación biológica de nitrógeno: Factores limitantes: En Fernández Balladares (Editor.). Ciencias y Medio Ambiente. Toledo. CSIC.

Grist, D. H. 1982. Arroz. México: Compañía Editorial Continental. S.A. C.V. México. Primera Edición en Español de la quinta edición en Inglés, oct. $1982,716 \mathrm{p}$.

Keeney, D. R. and D. W. Nelson. 1982. Nitrogeninorganic forms. In Methods of Soil Analysis. Part 2. Ed. By A.L. Page. R.H. Miller. D.R. Keeney. Madison. WI. EE.UU. ASA-SSSA. p. 643-698. (Series Agronomy No. 9).

Lacasta, C. y R. Meco. 2005. Manejo de agrosistemas de cereales. CSIC. Manual de agricultura ecológica. XI Jornadas Técnicas de la SEAE - Toledo.

Lampkin, N. 1998. Agricultura Ecológica. Madrid, Barcelona y México: Ediciones Mundi-Prensa. $725 \mathrm{p}$

Loveland, P., and J. Webb. 2003. Is there a critical level of organic matter in the agricultural soils of temperate regions: a review. Soil \& Tillage Research 70:1-18.

Maljean, F., F. Amlinger, C. G. Bannick, E. Favoino, I. Feix, I. Leifert, L. Marmo, R. Morris, C. Pallière, M. Robert, S. Siebert and F. Tittarelli. 2004. Land 
use practices in Europe. In: (Van Camp et al. Eds.) Reports of the Technical Working Groups Established under the Thematic Strategy for Soil Protection. (Luxembourg, EUR 21319) 872 p.

Ostergard, H., 2002. Characteristics of spring barley varieties for organic farming. Proceedings of the 1st. International symposium on organic seed production and plant breeding, Berlin.

Puig, M. H. 1979. Factores que afectan la respiración y la capacidad fijadora de nitrógeno de suelos con paja incorporada. INIA, Ministerio de Agricultura. Toledo, España. 246 p.

Reigosa, M., N. Pedrol y A. Sánchez. 2005. La Ecofisiología Vegetal una Ciencia de Síntesis. Cap. 13. Nitrógeno, Agricultura y Medio Ambiente. Ed. Thomson $1193 \mathrm{p}$.

Rodríguez, J., y H. Silva. 1984. Nitrógeno potencialmente mineralizable en Andisoles. Ciencia e Investigación Agraria 11:81-88.

Romanyà, J., y P. Rovira. 2007. Labile phosphorus forms in irrigated and rainfed semiarid Mediterranean grassy crops with long-term organic or conventional farming practices. European Journal of Agronomy 27:62-71.
Romanyà, J., P. Rovira y R. Vallejo. 2007. Análisis del carbono en los suelos agrícolas de España. Aspectos relevantes en relación a la reconversión a la agricultura ecológica en el ámbito mediterráneo. Revista Ecosistemas 16:1-8

Slanina, J., W. A. Lingerak y L. Bergman. 1976. A fast determination of nitrate in rain and surface waters by means of ultraviolet spectrophotometry. Z.Anal.Chem. 280, 365-368.

SPSS. 2005. Statistical Package for the Social Sciences. Version 14.0

Walkley, A. y I. Black. 1934. An examination of the Degtjareff method and a proposed modification of the chromic matter and a proposed modification of the chromic acid titration method. Soil Science 37:29-37. 